\title{
FAINT FIELD GALAXY COUNTS, COLORS, AND REDSHIFTS
}

\author{
DAVID C. KOO \\ University of California Observatories, Lick Observatory \\ Board of Studies in Astronomy and Astrophysics \\ University of California, Santa Cruz, CA 95064 USA
}

\begin{abstract}
This paper aims to provide the non-specialist a brief overview and major highlights in the rapidly changing research area of faint field galaxy evolution. Special emphasis is given to number counts, colors, and redshifts more recent than 1992 and their interpretation, both conventional and exotic. We close with previews of new directions that exploit sizes and velocity widths to explore the nature of faint blue galaxies, a puzzle that has defied solution for 15 years. The mystery continues.
\end{abstract}

\section{Brief History and Overview}

The interest in the evolution of faint galaxies was ignited by the watershed Yale Conference in 1977 when Kron presented the first credible evidence that field galaxies appeared numerous and quite blue. This result was a surprise, since the naive expectation is that fainter galaxies are redshifted and thus redder. Moreover, fainter galaxies should have number counts that declined rapidly relative to Euclidian slopes, since the volume per unit area drops with distance in Friedmann cosmologies. By the early 1980's, several groups had confirmed the large counts. There even appeared to be a consensus that the numerous, faint blue field galaxies could be explained by evolutionary models in which younger galaxies were undergoing more rapid star formation and were thus bluer than their descendents today. Furthermore, the large number counts could be understood because these galaxies were also brighter, more easily visible to higher redshifts $(z \sim 1-3)$, and thus counted within larger volumes per unit area of the sky.

By the late 1980's, confusion permeated the field, despite the confirmation to fainter magnitudes with CCD's of the older photographic results 


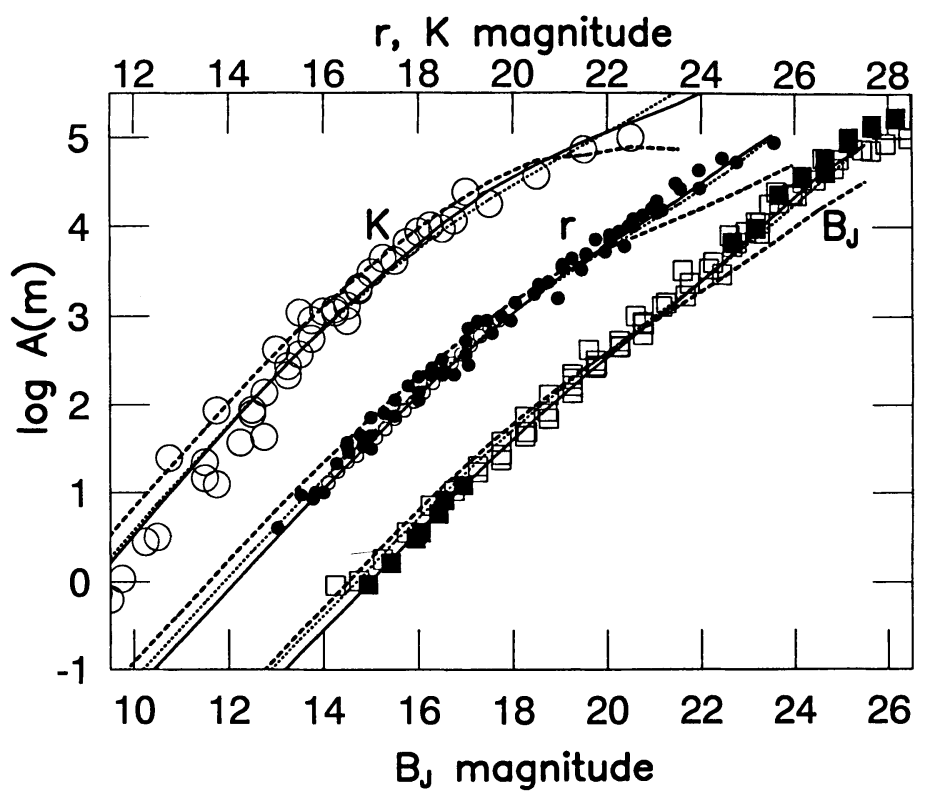

Figure 1. Log of the differential counts A (per mag per square degree) of faint field galaxies versus magnitude in the indicated bands from Gronwall and Koo (1995). $B_{\mathrm{J}}$ and Gunn $r$ band observations (open squares and solid circles) are compilations of data made by Koo \& Kron (1992); the infrared $K$ band counts (large open circles) are a compilation from Gardner et al. (1993). Solid squares show the bright $B_{\mathrm{J}}$ counts corrected for the systematic errors discovered by Metcalfe et al. (1994a) as well as the new faint CCD counts of Metcalfe et al. (1994b); small open circles show the new $r$ band counts of Weir (1994). The new mild evolution (with reddening) predictions are shown as solid curves while the equivalent best-fitting no-evolution predictions from Gronwall and Koo (1995) are shown as dotted curves. The short-dash curve shows a more typical no-evolution model, in this case from Rocca-Volmerange and Guiderdoni (1990).

of high counts and very blue colors. The key issue became whether the redshifts of faint galaxies are low or high. On the one hand, the Durham (UK) redshift surveys and cold dark matter (CDM) models supported the low side $(\mathrm{z}<1)$. On the other, Cowie and Lilly (1988) claimed to find a $\mathrm{z}=3.38$ field galaxy and a significant population of faint galaxies showing the Lyman break in a $3400 \AA$ filter, both results implying high redshifts $z$ $>2.7$. Together with numerous discoveries of high redshift $(z>2)$ radio galaxies, damped Lyman alpha lines, and QSO's, the high redshift end ( $z>$ 1) remained equally plausible. Today, consensus has not yet been reached in understanding the nature of faint blue galaxies, despite an enormous increase in data and theory. The following sections will try to convey the current status of the field and will provide an update on the key issues being addressed by researchers today. 

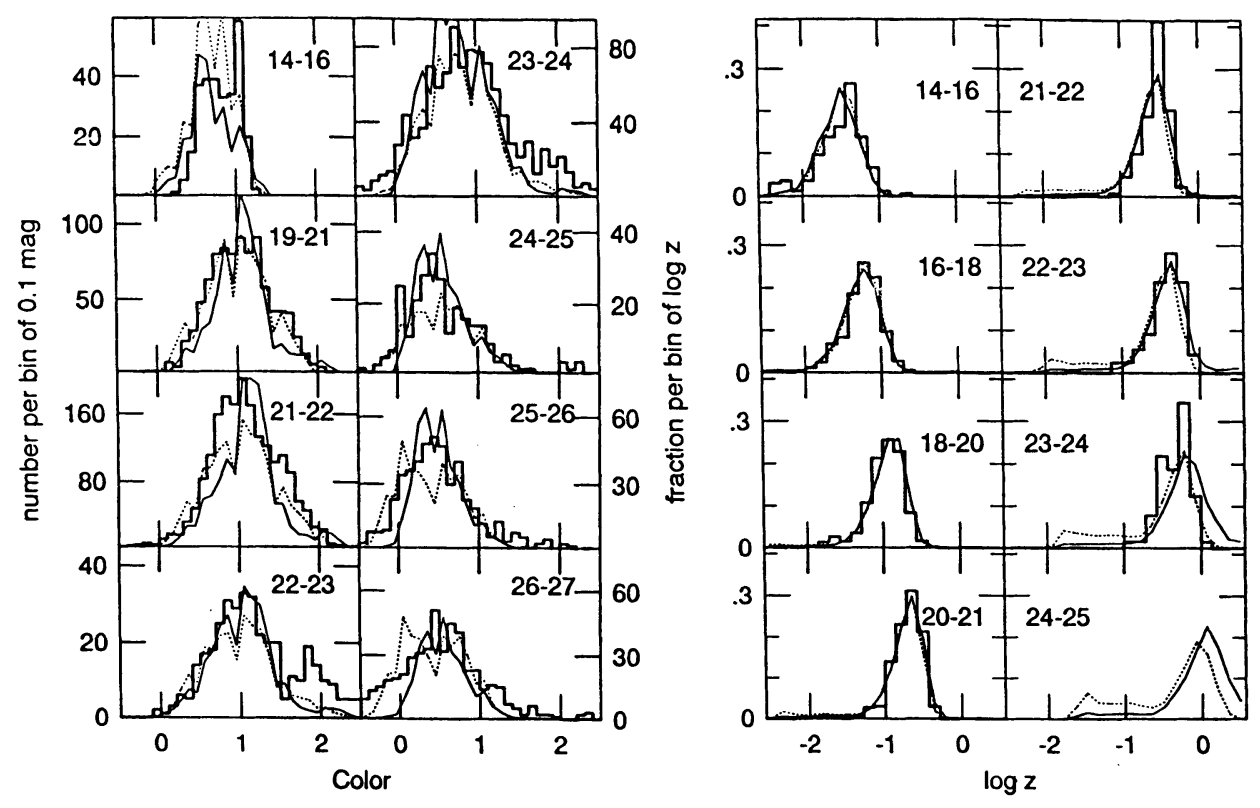

Figure 2. Left hand set of panels: Color distributions versus indicated magnitude intervals are shown as thick lines for the observations and thin solid lines for the mild evolution model predictions and dotted lines for the NE predictions from Gronwall and Koo (1995). The $B=14-16$ interval shows $B-V$ color while the other panels show $B_{\mathrm{J}}-R_{\mathrm{F}}$ color distributions in the given $B_{\mathrm{J}}$ magnitude intervals. Note the trend to bluer colors between galaxies in the $23<B_{J}<24$ panel to the $24<B_{J}<25$ panel. Right hand set of panels: Normalized histograms versus redshift $(\log z)$ for magnitude $\left(B_{\mathrm{J}}\right)$ intervals as indicated (Gronwall and Koo 1995). Bin size is 0.143 , since original bin size was 1.0 in $7 \log z$. The evolutionary model predictions are shown as thin solid lines while the NE model predictions are shown as dotted lines; observations shown as thick lines are compilations from different surveys.

\section{Observations and Theories}

The status of the observational evidence for evolution of faint field galaxies was reviewed by Koo and Kron (1992), to which the reader is referred for references prior to 1992 and further details. When compared to predictions of no-evolution (NE) models, these data showed number counts in the optical bands to exceed the expected counts by large factors, up to 5 to 15 times by $\mathrm{B} \sim 25$. In contrast, near-infrared counts in the $\mathrm{K}$ band $(2.2 \mu)$ appeared to be so low as to suggest a closed universe with little evolution. Figure 1 shows the current status of these faint counts in the blue $\left(B_{J}\right)$, red (Gunn $\mathrm{r}$ ), and near-IR (K). CCD photometry also confirmed that these faint (B > 20) galaxies were quite blue (Figure 2, left hand panels), but more impor- 
tantly, various new multi-object spectroscopic surveys from the Durham and Hawaii groups yielded redshift histograms that were surprisingly well fit by NE models, despite the count excess (Figure 2, right hand panels). In other words, there was no evidence for the high redshift $(z>1)$ tail expected from the "traditional" mild-evolution models of the 1980's. Further evidence for recent enhancement of star formation came from the strength and frequency of emission lines in these spectroscopic samples, while suggestions for a new population of objects at faint magnitudes were suggested by the low amplitudes found in the clustering (correlation function) among faint galaxies.

To explain these data, a number of new ideas have been proposed. These can be roughly divided into two camps. One set of theories is "exotic" in the sense that one or more basic assumptions of the traditional models, such as galaxy number conservation, is modified and predominately affects the observations. The exotic ideas include proposals that: dwarf galaxies today were much brighter in the recent past; galaxies have been undergoing extensive mergers in the recent past; the universe has a non-zero cosmological constant; or a new population of galaxies at moderate redshifts have disappeared by today. The reader is referred to Lilly (1993) for references and further details.

The other "conservative" explanations propose a combination of uncertainties in the observations or local galaxy properties to explain most of the faint galaxy data, leaving only minor extraordinary components. The conservative explanations include the possibility that the faint end slope of the local luminosity function (LF) of galaxies is steeper than commonly adopted in models (Driver et al. 1994, Marzke et al. 1994, Koo et al. 1993, Treyer \&Silk 1994); that low surface brightness galaxies detected in faint samples have been systematically missed in more local surveys (McGaugh 1994, Ferguson and McGaugh 1994); that the local LFs as a function of color are poorly known (Shanks 1990, Metcalfe et al. 1991, Koo et al. 1993); that the local density remains uncertain due to large scale structure (Picard 1991) or to systematic magnitude errors (Metcalfe et al. 1994a, Weir 1994); and that some traditional models, with minor modifications, are good fits (Yoshii and Peterson 1991, Wang 1991, Gronwall and Koo 1995). Figures 1 and 2 show that new traditional models can provide good fits to the counts, colors, and redshifts and thus exotic explanations may not be necessary.

\section{Recent Highlights}

The observations have continued to expand dramatically since the review of Koo and Kron (1992). Besides the numerous aforementioned references supporting the conservative view, optical counts and colors to very faint 


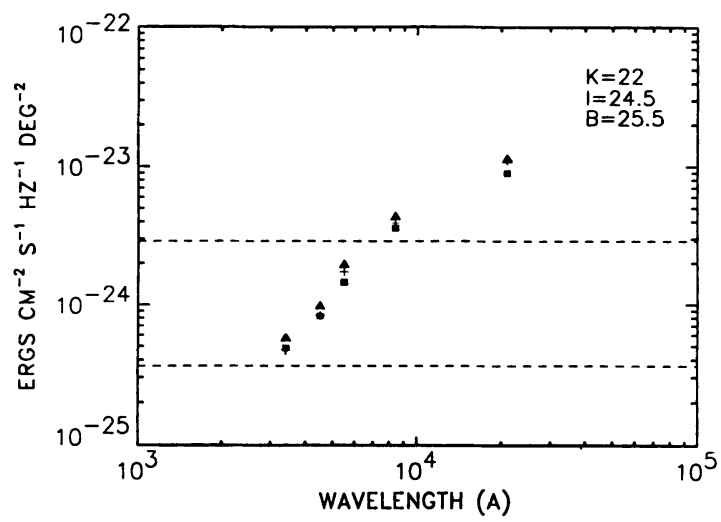

Figure 3. Extragalactic background light versus wavelength as copied from figure by Cowie et al. (1994). The triangles show the light from $\mathrm{K}<22$ galaxies; the crosses from I $<24.5$ galaxies; and the boxes from $B<25.5$ galaxies. The dashed lines bracket the range of blue background light predicted from star formation needed to produce the heavy elements found in luminous matter today.

limits have been measured by several new, independent groups, but whether a turnover exists in the steep optical number counts is not yet settled (Steidel \& Hamilton 1993, Metcalfe et al. 1994b). Near-IR (K) counts have been improved at both the bright and faint limits (Gardner et al. 1993, Soifer et al. 1994, Djorgovski et al. 1994, Cowie et al. 1994, McLeod et al. 1994). More importantly, several new redshift surveys to various depths and selected by optical or near-IR bands have been completed (Lilly 1993, Colless et al. 1993, Tresse et al. 1993, Glazebrook and Ellis 1994, Songaila et al. 1994). Soon to be published will be the Canada-France Redshift Survey with its impressive sample of 1000 objects to a limit of $\mathrm{I} \sim 22$ (B 24.5). Of most relevance to the topic of this symposium is the very recent estimate of the extragalactic background light from deep optical and near-IR counts by Cowie et al. (1994) and shown in Figure 3. Their main conclusion from this figure is that the background has a red spectrum, but its blue part can be plausibly explained by the star formation needed to create the heavy elements we see in luminous matter today.

The scientific conclusions from all these new observations remain controversial. Several groups claim that the traditional luminosity evolution models are excluded because no high redshift tail is found in the deepest redshift surveys (Broadhurst et al. 1992, Glazebrook and Ellis 1994). Indeed, the trend is so strong that there may actually be a lack of redshift $z \sim 1$ galaxies compared to even the no-evolution estimates (Colless et al. 1993, Koo et al. 1993, Songaila et al. 1994). This could result from galaxies having been in smaller subunits prior to merging. One area of apparent consensus is that the $\mathrm{K}$ band counts no longer appear to be incompatible 
with the optical blue counts (Gardner et al. 1993, Koo et al. 1993) when the bluer colors of fainter galaxies are included in the models. Several efforts have recently been directed to the explicit determination of the LF of galaxies as a function of redshift. The trends appear consistent among the work of three groups (Eales 1993, Lonsdale \& Chokshi 1993, Colless 1994) - the LF appears to have a steeper faint end slope and/or a higher normalization $\left(\Phi^{*}\right)$ at higher redshifts when compared to local estimates. On the other hand, analysis of galaxies associated with foreground Mg II absorption lines seen in background QSOs indicate no evidence for density, luminosity, or color evolution (Steidel, Dickinson, \& Persson 1994). Nor do the observations of emission line galaxies show any evolution (Boroson et al. 1993). A key question to be settled is whether a large exotic component at the level of $200 \%$ or more is needed (Lilly 1993, Babul \& Rees 1992, Broadhurst et al. 1992) or whether, as shown by the good fits in Figures 1 and 2, a smaller amount at the level of 10's of percent or less will suffice, if the more conservative view is closer to the truth (Gronwall and Koo 1995).

\section{New Directions}

Several programs will soon make major contributions to our understanding of faint galaxies. Of particular note is the high spatial resolution now available with the refurbished Hubble Space Telescope. Analyses of such images are yielding sizes, surface brightnesses, morphologies, color gradients, signatures of nuclear starbursts or interactions, etc., for faint field galaxies on scales of $\mathrm{kpc}$ for redshifts beyond $\mathrm{z} \sim 0.2$. Several different groups are pursuing this approach to study faint field galaxies; the reader is referred to the contribution in this volume by R. Griffiths who describes some of the results from the Medium Deep Survey, an HST Key Project.

Another direction is the extraction of velocity widths or rotation curves of distant galaxies. Combined with sizes and inclinations from HST data, masses can be derived. Initial efforts in these directions have already been undertaken by several independent groups (Franx et al. 1992, Vogt et al. 1993, Colless 1994, Koo et al. 1995). Figure 4 gives an example from using the echelle spectrograph on the Keck 10-m telescope; the wide range in velocity widths for a sample of otherwise similarly luminous galaxies supports the need for kinematics as a powerful new tool to study the nature of distant galaxies. With improved signal-to-noise spectra, spectral signatures of age and metallicity from emission and absorption lines, rather than just redshifts, become feasible for analysis. Initial forays using emission lines are beginning to be published (Smetanka 1993, Tresse et al. 1994).

We close with what may not be obvious, namely the need for improved data on the properties of nearby galaxies. In a few years, the Sloan Digital 


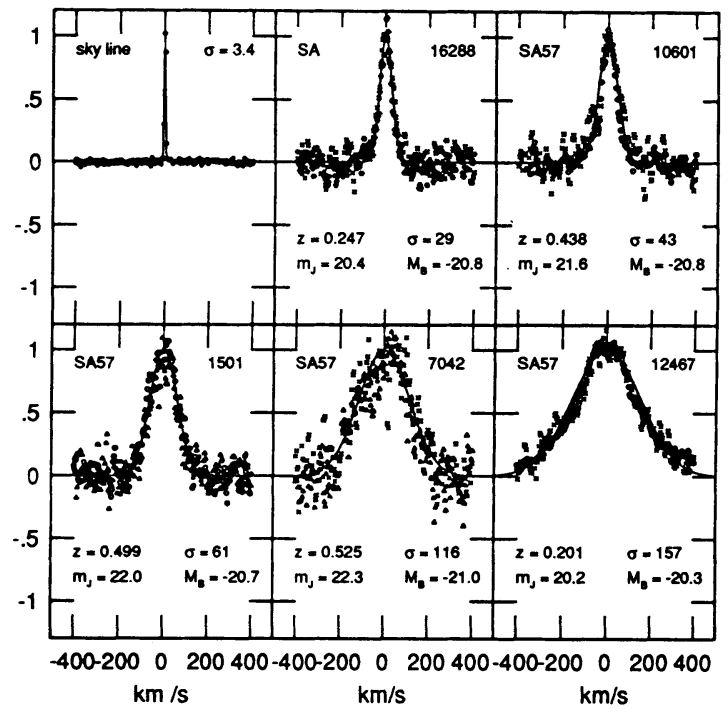

Figure 4. Velocity widths for sample of five compact blue galaxies (Koo et al. 1995). The upper left panel shows the instrumental profile from a typical night sky line for the echelle (HIRES) on the Keck 10-m telescope. The solid line in each panel is the best fit Gaussian curve. The name of each object is given, as is the redshift (z), the sigma of the fitted Gaussian in $\mathrm{km}-\mathrm{s}^{-1}$, the apparent blue $\left(B_{J}\right)$ magnitude, and the blue luminosity $\left(M_{B}\right)$ assuming a Hubble constant of $50 \mathrm{~km}-\mathrm{s}^{-1}-\mathrm{Mpc}^{-1}$ and $q_{0}=0.1$.

Sky Survey will provide a quantum leap in such vital information (Gunn \& Knapp 1992). This survey will use a dedicated 2.5 -m telescope to image 10,000 square degrees of sky in 5 optical bands (roughly UBVRI) to a limit of $B \sim 24$, as well as to secure spectra of about 1,000,000 field galaxies to $\mathrm{B} \sim 19.5$. If the progress since 1992 is any indication, this review will be out of date by the time it is published. Such rapid changes are regarded by some as a sign of confusion within the field; others consider such instability as a sign of a healthy and exciting research area. The mystery of faint blue galaxies has withstood the onslaught of many dedicated observers and theorists for over 15 years; the mystery is not likely to vanish immediately.

\section{Acknowledgements}

I wish to thank L. Cowie, K. Glazebrook, C. Gronwall, R. Guzmán, S. Lilly, B. McLeod, and A. Phillips for their inputs into this review and the organizers for their invitation. This work was supported by NSF grants AST 88-58203 and AST 91-20005. 


\section{References}

Babul, A., \& Rees, M. J. 1992, MNRAS, 255, 346

Boroson, T. A., Salzer, J. J., \& Trotter, A. 1993, ApJ, 412, 524

Broadhurst, T. J., Ellis, R. S., \& Glazebrook, K. 1992, Nature, 355, 55

Charlot, S., \& Bruzual A., G. 1991, ApJ, 367, 126

Colless, M., Ellis, R. S., Broadhurst, T. J., Taylor, K., \& Peterson, B. A. 1993, MNRAS, 261,19

Colless, M. 1994, in Wide Field Spectroscopy and the Distant Universe, eds. S. Maddox and A. Aragón-Salamanca (World Scientific Pub.)

Cowie, L. L., Songaila, A., \& Hu, E. M. 1991, Nature, 354, 460

Cowie, L. L., Gardner, J. P., Hu, E. M., Hodapp, K. W., \& Wainscoat, R. J. 1994, ApJ, in press.

Djorgovski, S., Soifer, B. T., Pahre, M. A., Larkin, J., et al. 1994, ApJ, in press

Driver, S. P., Phillips, S., Davies, J. I., Morgan, I., \& Disney, M. J. 1994, MNRAS, 266, 155

Eales, S. 1993, ApJ, 404, 51

Ferguson, H. C., \& McGaugh, S. S. 1994, ApJ, in press

Franx, M. 1993, ApJ, 407, L5

Gardner, J. P., Cowie, L. L., \& Wainscoat, R. J. 1993, ApJ, 415, L9

Glazebrook, K., \& Ellis, R. S. 1994, preprint

Gronwall, C., \& Koo, D. C. 1995, ApJ, in press.

Gunn, J. E., \& Knapp, G. R. 1993, A. S. P. Conf. Ser. No. 43, 267

Koo, D. C., Gronwall, C., \& Bruzual A., G. 1993, ApJ, 415, L21

Koo, D. C., Guzmán, R., Faber, S. M., Illingworth, G. D., Bershady, M. A., Kron, R. G., \& Takamiya, M. 1995, ApJ, submitted.

Koo, D. C., \& Kron, R. G. 1992, ARAA, 30, 613

Lilly, S. J. 1993, ApJ, 411, 501

Lilly, S. J., Cowie, L. L., \& Gardner, J. P. 1991, ApJ, 369, 79

Lonsdale, C. J., \& Chokshi, A. 1993, AJ, 105, 1333

Marzke, R. O., Huchra, J. P., \& Geller, M. J. 1994, ApJ, in press

McGaugh, S. S. 1994, Nature, 367, 568

McLeod, B. A., Bernstein, G. M., Rieke, M. J., Tollestrup, E. V., \& Fazio, G. G. 1994, ApJS, in press

Metcalfe, N., Fong, R., \& Shanks, T. 1994a, MNRAS, submitted

Metcalfe, N., Shanks, T., Fong, R., \& Jones, L. R. 1991, MNRAS, 249, 498

Metcalfe, N., Shanks, T., Fong, R., \& Roche, N. 1994b, MNRAS, submitted

Picard, A. 1991, ApJ, 368, L11

Rocca-Volmerange, B., \& Guiderdoni, B. 1990, MNRAS, 247, 166

Shanks, T. 1990, in IAU Symp. 139, The Galactic and Extragalactic Background Radiation, eds. S. Bowyer \& C. Leinert, (Kluwer: Dordrecht), p. 269

Smetanka, J. J. 1993, Ph.D. thesis, Univ. of Chicago

Soifer, B. T., et al. 1994, ApJ, 420, L1

Songaila, A., Cowie, L. L., Hu, E. M., \& Gardner, J. P. 1994, ApJ, in press.

Steidel, C. C., Dickinson, M., \& Persson, S. E. 1994, ApJ, in press

Steidel, C. C., \& Hamilton, D. 1993, AJ, 105, 2017

Tresse, L., Hammer, F., LeFevre, O., \& Proust, D. 1993, Astron. \& Astrophys., 277, 53

Tresse, L., Rola, C., Hammer, F., \& Stasinska, G. 1994, in Wide Field Spectroscopy and the Distant Universe, eds. S. Maddox and A. Aragón-Salamanca (World Sci. Pub.)

Treyer, \& Silk, J. 1994, ApJ, in press

Vogt, N. P., Herter, T., Haynes, M. P., and Courteau, S. 1993, ApJ, 415, L95

Wang, B. 1991, ApJ, 383, L37

Weir, N. 1994, Ph.D. thesis, California Institute of Technology

Yoshii, Y., \& Peterson, B. A. 1991, ApJ, 372, 8 